\title{
Inklusion/Exklusion
}

\section{Zur Ambivalenz des Hauses bei Kafka und Musil}

\author{
Alexander Honold
}

\section{Das Haus und der Raum}

Unter einem sicheren Dach, auf einem befestigten Boden und sich zwischen mehr oder minder vier Wänden zu befinden, gibt dem Menschen ein existentielles Grundvertrauen, das nicht auf Kosten einer weiter ausgreifenden räumlichen Orientierung gehen muss. Im Gegenteil; nicht erst seit Martin Heideggers Essay »Bauen Wohnen Denken« ist dargelegt worden (vgl. Heidegger (2000 [1951]), dass die gebaute Ordnung des Hauses nicht wirklich den Ausschluss von Welt bedeutet, sondern deren zeichenhafte Reproduktion. ${ }^{1}$ Im Fußboden liegt - pars pro toto - eine Inkorporation des Erdbodens vor, die Wände umreißen die Blickdirektion in alle vier Himmelsrichtungen, auch wenn sie realiter durch ihre stoffliche Zusammensetzung die Sichtmöglichkeiten mehr oder minder stark reduzieren. Und im Anblick der Zimmerdecke und des Dachaufbaus ist die Überwölbung des irdischen Daseins durch den Himmel zu einem Gegenstand baulicher Rekonstruktion geworden. So ließe sich sagen, dass in den Grundformen des Hauses, und dies vermutlich für historisch und kulturell höchst verschiedene Ausprägungen und Bauformen gleichermaßen, eine konstruktive Nachahmung der anthropologischen WeltOrientierung vorliegt, durch die der Mensch sich einen selbstgesetzten Raum innerhalb des natürlichen und kosmischen Raumes verschafft.

1। Die kulturelle Replikation der menschlichen Naturverhältnisse in per se ambivalenten Gründungsakten und Behausungstechniken untersucht Saskia Haag (vgl. Haag 2012). 
Inklusion und Exklusion der Außenwelt stellen folglich im Modell des Hauses keinen dichotomisch strukturierten Gegensatz dar, sondern vielmehr ein Komplementärverhältnis. Die Inklusion des gesamten kosmischen Gefüges in die vier Wände eines bescheidenen Zimmers kann deshalb als symbolische Form der Teilhabe gelingen, weil sie mit der Exklusion unkontrolliert einwirkender Naturkräfte verbunden ist. Einerseits also bleibt das physikalische Ausgerichtetsein auf die Grundachse der Vertikale und die Koordinaten des Rechts-Links- sowie Hinten-Vorn-Richtungskreuzes, mit dem sich der Mensch die Vorteile seines vielbeschworenen aufrechten Gangs zunutze macht, als elementares Formparadigma sämtlicher Raumvorstellungen auch in den baulichen Behältnissen aller Art grundsätzlich erhalten und als Bewertungssystem weiterhin virulent. Andererseits aber liegt der Sinn dieses sekundär geschaffenen, baulich umgrenzten Raumes, wie ihn Häuser, Wohnungen und Zimmer darstellen, zweifellos auch und gerade in den damit verbundenen AusschlussMöglichkeiten.

Der von dem Philosophen Hans Blumenberg so genannte »Absolutismus der Wirklichkeit« (Blumenberg ${ }^{6} 2001$ [1979]: 10) beschreibt jenen Aspekt der conditio humana, der sich aus der Preisgegebenheit gegenüber etwelchen Gefahren ableitet, die aus verschiedensten Richtungen herannahen können; diesem ubiquitären Gefahrenpotential begegnet nichts derart wirkungsvoll wie die zivilisatorischen Errungenschaften des Bauens. Hier nämlich lernt der Mensch nicht nur, sich selbst eine Zone der Hegung und des Schutzes gegen außen zu verschaffen; er macht sich vor allem mit den Mechanismen vertraut, dieses primäre Schutzbedürfnis wiederum zu delegieren an das bereits Vorhandene und institutionell Abgesicherte. Wo Boden, Dach und Wände errichtet und stabile Raumbehältnisse geschaffen wurden, kann die existentielle Grundsorge, wie sie entwicklungsgeschichtlich durch ubiquitär drohende Gefahrenlagen motiviert war, an das etablierte Daseinsmodell des Wohnens in geschützten Räumen delegiert werden. Noch unser Reflex, in größeren öffentlichen Räumen nach Möglichkeit mit dem Rücken nahe an einer der Wände zu sitzen, leitet sich von derartigen Strategien der Eindämmung jenes besagten Absolutismus der Wirklichkeit her. Man schließt damit aus, dass Unerwartetes aus jenen Richtungen kommen könnte, die man selbst nicht gut im Blick hat. Diese Form der Delegation von Gefahrenabwehr stellt neben Aspekten der Bequemlichkeit und der Witterungsresistenz eine der wesentlichen Triebkräfte des Bauens dar. 
Im Kulturkonzept des Wohnens als einer dauerhaften Nutzung von umbauter Innenräumlichkeit spielt neben den benannten Faktoren des Schutzes und der Witterungsunabhängigkeit freilich auch die Zeitdimension noch eine entscheidende Rolle. Wohnen und Gewohnheiten sind nicht nur etymologisch eng verwandt, sondern auch sachlich aufeinander bezogen. Der bewohnte Raum des befestigten Hauses, der umgrenzten Wohnung oder des abschließbaren eigenen Zimmers ist diejenige Bezugsgröße des Handelns, Fühlens und Denkens, in der man sich über die sonstigen Raumverhältnisse und das komplexe System natürlicher und technisch geschaffener spatialer Strukturen nicht mehr allzu viele Gedanken machen muss. Meistens gelingt es uns nicht einmal mehr, in innenräumlichen Bezügen ein klares Bewusstsein zur Fixierung der vier Himmelsrichtungen aufrecht zu erhalten. Das ist Symptom des hohen Grades, in dem die primäre räumliche Daseinsvorsorge an das architektonische Werk der zweiten Natur hat delegiert werden können. Wir brauchen die elementaren Raumkoordinaten nicht mehr ständig selbst zu erfassen und hinsichtlich ihrer Stabilität und ihrer jeweiligen strategischen Relevanz zu überprüfen; in hohem und kaum mehr wirklich mitreflektiertem Maße wird dies für uns durch die nahezu durchgängigen architektonischen Konstruktionen unserer alltäglichen Lebenswelt übernommen. Institutionell geregelt, nämlich an Eingangsportale, Zugangscodes und Aufnahmerituale delegiert, sind nahezu sämtliche basalen Prozesse der In- und der Exklusion und die mit ihnen verbundenen Unterscheidungsoperationen. Erst wo innerhalb des institutionellen Gefüges eine Störung oder ein Krisenfall eintritt, werden die eingespielten Mechanismen überhaupt als solche wieder sichtbar. Und gerade im Ausnahme- oder Störungsfall fällt dann auch die Notwendigkeit der in- und exkludierenden Wohnstrategien wieder auf das eigenverantwortliche und spontane Handeln individueller Protagonisten zurück.

Solche Rückfälle in ein okkasionelles und improvisiertes Aushandeln der Grenzen des Hauses zählen nicht von ungefähr in zahlreichen literarischen Texten zu den privilegierten Handlungskonstellationen, wie ja überhaupt der Ausnahmefall erzähltechnisch eine weit größere Ergiebigkeit besitzt als die gewohnheitsmäßig eingespurten Routine-Abläufe. Auch innerhalb des symbolischen kulturellen Systems der Literatur fungiert das Haus als ein räumliches Grundmodell topologischen Denkens; es definiert die Mensch-Natur-Beziehung durch den Gegensatz von Basis und Überbau, reguliert die Sozialverhältnisse anhand der Unterschei- 
dung von öffentlich und privat und stiftet die generationenübergreifende Kontinuität eines Erbschafts- und Traditionszusammenhanges. An zwei Erzähltexten werden die Implikationen und Spielräume dieser oikologischen Raummodellierung exemplarisch nachgezeichnet. In Kafkas Der Bau (1924) wird die verhängnisvolle Dualität von Abschottung und Projektion zur Triebkraft eines paranoid perfektionierten Weltentwurfs; für Robert Musils Der Mann ohne Eigenschaften (1930) wird das Domizil des Protagonisten zur symbolischen Synopse eines eklektischen Zeitalters. Dabei zeigt sich die in den besprochenen Texten jeweils etablierte Raumsemantik auch abhängig von den Dispositionen der jeweiligen Figurenperspektive und Erzählstrategie.

\section{Kafka und die Paranoia}

»Ich habe den Bau eingerichtet und er scheint wohlgelungen «. ${ }^{2}$ Auf den ersten Blick gibt der unbekannte Ich-Erzähler in Franz Kafkas aus dem Nachlass überlieferten Erzählung Der Bau von einem geglückten architektonischen Schaffensakt Nachricht. Fast wie im biblischen Schöpfungsbericht schaut der Urheber auf sein Werk zurück und befindet es für wohlgelungen. Doch werden Meisterstolz und Zuversicht in dieser Erzählung bald kaum noch die Oberhand behalten; vielmehr weckt die Beschäftigung des selbst berichtenden Akteurs mit der Anlage und den Eigenschaften seines Bauwerkes zunehmende Zweifel und Ängste, bis schließlich der Baumeister sich des Erfolgs seiner Kunst keineswegs mehr gewiss ist und sie sogar für den allergrößten Fehler seines Lebens zu halten beginnt.

Nicht mehr Herr im eigenen Hause zu sein, diese von Freud beschriebene Grundbedingung der modernen Psyche ist in der Auseinandersetzung des kleinen Endbewohners mit seinen mutmaßlichen Feinden zur ganz wörtlich genommenen Wohnsituation geworden. »Hier gilt auch nicht daß man in seinem Haus ist, vielmehr ist man in ihrem Haus « (Kafka 1994: 167). Freimütig kommt der Protagonist in der Folge auf immer mehr kleine und größere Schwachstellen der selbstgeschaffenen unterir-

2 | Der Titel Der Bau ist eine editorische Konstruktion ex post; daher wird die Erzählung bibliographisch unter ihrem Incipit "Ich habe den Bau eingerichtet..." geführt (vgl. Kafka 1994: 165). 
dischen Anlage zu sprechen; Fehler, vor welchen er einerseits die Augen gerne verschließen würde, auf die er andererseits aber mit selbstquälerischer Beharrlichkeit immer wieder zu sprechen kommt. Ganz offen bekennt er, dass er »nicht immer einen Mangel des Baues in Augenschein nehmen« wolle, »wenn dieser Mangel schon in meinem Bewußtsein nur allzusehr rumort« (Kafka 1994: 175).

Weder dem eigenen Handwerk noch der vernünftigen Urteilsfähigkeit ist in diesem Werk zu trauen, wenn selbst die unmittelbaren Sinnesdaten schon nur mehr trügerische Vorstellungen von den Verhältnissen der Wirklichkeit liefern. In der beschreibungsintensiven Erzählprosa Franz Kafkas finden die durch Institutionen und Medien induzierten Deformationen der sinnlichen Lebenswelt einen genauen und unbestechlichen Chronisten; so berichten seine Erzählungen ebenso von einem nach Verlassen der Eisenbahn fortwirkenden Gefühl der Seekrankheit wie von dem Zurückschaudern vor dem Eintritt in die machtgestützte Diskurswelt des Gesetzes, des Gerichts oder des Schlosses. Ein permanentes »Zischen« oder »Pfeifen« (Kafka 1994: 189) beklagt jenes wundersame Tier (vermutlich ein Maulwurf?), das in Kafkas Erzählung Der Bau aus dem Inneren eines selbstangelegten unterirdischen Höhlensystems rapportiert. Hat man Der Bau einerseits als Reflexion auf die Weltkriegs-Erfahrungen des Stellungs- und Grabenkrieges bezogen (vgl. Kittler 1990), so ist ebenfalls mit plausiblen Argumenten auch schon das setting dieses bizarren Maulwurf-Interieurs als dasjenige einer Expedition in den menschlichen Gehörgang verstanden worden (vgl. Encke 2006 u. Steiner 2012).

Eine Verarbeitung der Fronterfahrungen des »Grabenkriegs«, so hatte Wolf Kittler argumentiert, kann bei Kafka auf zumindest indirektem Wege durchaus vorliegen, vermittelt über einen einschlägigen Bericht Bernhard Kellermanns (Der Krieg unter der Erde, Juli 1915) ${ }^{3}$ sowie über die persönlichen Erzählungen des Schwagers Josef Pollak bei einem Fronturlaub im Herbst $1914^{4}$ und über die Nachbildung eines Schützengrabens

3 | Kellermanns Bericht, so Kittler, befand sich in einer Sammelausgabe in Kafkas Bibliothek, was die mutmaßliche Referenz auf diesen Text bestätigt (vgl. Kittler 1990: 290 ff.).

4 | Kittler 1990: 292 u. Encke 2006: 139. 
für die Prager Bevölkerung im November $1915 .{ }^{5}$ Doch erweist sich gerade im Hinblick auf die ästhetischen Determinanten eines grabenkriegsähnlichen Belagerungszustandes der Umstand als sprechend, dass Kafka die Lebenswelt und den Aufmerksamkeitsradius des sich in Erdgänge eingrabenden Tieres ausschließlich auf Tastsinn und Hörvermögen bezieht; es handelt sich bei dem Text, so Julia Encke, um die existentiell radikale Beschreibung einer »Hörsituation« (Encke 2006: 133), bei der die kriegstypische Anmutung des Grabensystems mit der labyrinthischen Ordnung des menschlichen Gehörgangs überblendet wurde. ${ }^{6}$ Demzufolge ist Der Bau als textuelle Verarbeitung »kein Symptom des Krieges«, sondern »eine Erzählung, die die im Krieg relevant gewordene Wahrnehmungssituation weiterschreibt« (ebd.: 141).

Das erzählende Tier verbringt seine Zeit, da es über das alarmierende Geräusch eines möglicherweise nahenden Eindringlings und mutmaßlichen Feindes in höchste Angst versetzt ist, statt im tiefen Inneren der Anlage nun zunehmend »an der Grenze [...] zwischen Innen- und Außenwelt« (Steiner 2012: 160), tastet sich »horchend an den Wänden« entlang zum »Ort der Störung « (Kafka 1994: 188). Dann aber macht dieser Höhlengangbewohner die Entdeckung, dass der Entstehungsort und damit die Quelle des beunruhigenden Geräuschs auch bei gründlichster Suche gar nicht lokalisierbar ist. »Ich komme«, so stellt das Tier des Baus nämlich fest, »gar nicht dem Ort des Geräusches näher, immer klingt es unverändert dünn in regelmäßigen Pausen, einmal wie Zischen, einmal eher wie Pfeifen« (ebd.: 189). Grund dieser Verwirrung könnte sein, dass der Feind, den das Tier in seinen Bau eingedrungen wähnt, tückischerweise seine Position häufig wechselt und geradezu überall zu lauern scheint. Eine andere mögliche Erklärung aber, so verrät der Text, würde sich eröffnen mithilfe der Vermutung, dass es »das eigene Blut im Ohr« (ebd.: 197) sei, welches die irritierenden Geräusche verursacht. Liegt aber die Quelle des Pfeifens oder Zischens im Beobachter selbst, so lässt sich die Schwierigkeit, dem Störgeräusch einen präzisen Ort und ein klar mar-

5 | Prager Tagblatt v. 19. November 1915 (vgl. Encke 2006: 140). Kafka notiert den "Anblick der Ameisenbewegung des Publikums vor dem Schützengraben und in inm “ in einem Tagebucheintrag vom 6. November 1915 (Kafka 2002: 772). 6 | "Kafkas Bau, der in der Erzählung ausdrücklich als eine 'Höhlung، bezeichnet wird [...], evoziert nicht zuletzt diesen anatomischen Bau des Ohrs" (Encke 2006: 149). 
kiertes Gegenüber zuzuweisen, leicht durch eine kohärente Lesart auflösen: »Das Pfeifen und Zischen im Ohr des Tieres zeigt [...] den Lärm der selbstgeschaffenen Welt an« (Steiner 2012: 161).

Wie schon in der Parabel Vor dem Gesetz und letztlich auch in Kafkas Romanen insgesamt ist es wiederum der Protagonist selbst, der unwillentlich über Art und Umfang der sich ihm in den Weg stellenden Schwierigkeiten entscheidet. Nimmt man diesen konstitutiven Anteil des Protagonisten an dem innerhalb des Baus überall vernommenen bedrohlichen Höreindruck auf, so kann diese kulturgeschichtlich-aisthetische Rekonstruktion ohne Weiteres mit einer eher schreibästhetisch ausgerichteten Lektüre des Textes (wie sie etwa Bettine Menke skizzierte; vgl. Menke 1992) verbunden werden. Evidenterweise ist der Vorgang des Schreibens demjenigen des Grabens eng verwandt, um genau zu sein: sogar metonymisch assoziiert.

Kafkas fragmentarisch endende Erzählung vom Maulwurf, der in dem eigenen weitläufigen Sicherheitstrakt zunehmend die Welt seines gefährlichsten Feindes erkennen muss, weist einen Textbau auf, der auf dramatische, vielleicht letztlich tragische Weise die Aporie literarischer Produktionsästhetik inszeniert: das Gefangensein im eigenen Werk. Was der Erzähler-Protagonist innerhalb der eigenen Gänge zu hören bekommt, ist stets ein Echo seiner selbst, oder anders formuliert: eine Resonanzverstärkung seiner eigenen, hoch sensibilisierten Empfangsvorrichtungen. Denn Der Bau stellt nicht nur eine topologische Modell-Vergrößerung des Gehörganges und seiner dunklen Windungen dar, er reflektiert zugleich auch (nämlich als Musterfall eines von Kunstwillen erschaffenen Artefakts) die autopoietische Spannung zwischen Weltrezeption und Werkprojektion. Der Tinnitus, den man mit Uwe C. Steiner als einen heimlichen Helden Kafkas in dessen vielfach vermessenem »Hauptquartier des Lärms« (Steiner 2012: 159) ausmachen kann, ist symptomaler Ausdruck eines demiurgischen Dilemmas entgleister Schöpfungsprojekte. Neu und unerhört an der von Kafka gestalteten Dialektik der Subjektion ist, dass hier die selbstgeschaffene Schuldordnung der Protagonisten nicht mehr vorrangig mit den gängigen optischen Trugmetaphern der Illusion oder Projektion beschrieben wird, sondern eine primär architektonische Vergegenwärtigung erfährt, die sich an dem Außen-Innen-Resonanzverhältnis des Hörens orientiert.

In Kafkas Erzählprosa haben wir es mit einer Art von Texten zu tun, deren unreliability im Unterschied zum späten 19. Jahrhundert keine fi- 
gurenpsychologisch motivierte Grundlage mehr hat (sodass die Unzuverlässigkeit als ein Instrument der Charakterzeichnung werkintentional eingeordnet werden könnte), sondern nur mehr mittels einer symptomatologisch orientierten Lektüre zu entschlüsseln ist. Hier ist Der Bau in mehrfacher Hinsicht aufschlussreich; zum Ersten, da er die unaufhebbare Verstrickung des Bauens und des Wohnens ineinander zu einem existentiellen Befund erhebt - es gibt kein Sich-Einrichten im fertigen Bau, sondern nur permanente Umarbeitungen, die niemals fertig werden; zum Zweiten, indem Kafka hier die Paradoxien der Inklusion und der Exklusion dergestalt auf die Spitze treibt, dass jede Form der Abschottung nach außen sowohl als Verwirklichung von Sicherheitsmaßnahmen wie auch als Irritationsquelle neuer Unsicherheiten und Ängste erscheint, weil der Feind ja womöglich schon längst im Inneren des weitverzweigten labyrinthischen Gängesystems weilt oder aber durch die aufwendigen Baumaßnahmen überhaupt erst auf den Plan gerufen worden ist. Und drittens schließlich beginnen nicht mehr nur Abschottung und Risiko im Zuge der sich immer weiter perfektionierenden Baulogik zunehmend ineinander überzugehen, sondern auch die Grenzen des bauenden Akteurs selbst scheinen sich immer mehr aufzulösen und tendenziell in diejenigen des eigenen Gebäuderaumes zu diffundieren, sodass letztlich sogar zwischen der Architektur einer unterirdischen Stollenanlage und dem nur mentalen Konstrukt der mitgeteilten innerpsychischen Gedankengänge keine substanzielle Differenz mehr ausgemacht werden kann.

Wie - um mit einem wandlungsfähigen Leitwort des Textes zu sprechen - für die Antriebskräfte des Baus nicht nur der Faktor der List ausschlaggebend wurde, sondern ebenso sehr jene der Lust und schließlich der Last, sodass die List des Bauens, die Lust des Bauens und die Last des Bauens sich zu einer mehrfarbig schillernden Leitchiffre überlagern, so gilt von den hier ausführlich beschriebenen Anstrengungen des Bauens, Grabens und Schichtens insgesamt ebenfalls, dass in ihnen die wunschund die angstbesetzten Antriebskräfte eigentlich kaum mehr auseinanderzuhalten sind. $\mathrm{Ob}$ sich wirklich ein fremdes und großes, deshalb wohl unbedingt auch gefährliches Tier in den Bau eingeschlichen habe, wie der alarmierte Protagonist des Geschehens immer wieder mutmaßt, kann von der bloß mentalen Konstruktion einer solchen Gefahr innerhalb des entsprechenden paranoiden Systems gar nicht mehr unterschieden werden. 
Paranoia stellt sich innerhalb des Textes deshalb als pansemiotische Wahrnehmungs- und Deutungsspirale dar, die sowohl beunruhigende wie entwarnende Außensignale gleichermaßen zur Selbstverstärkung eines panischen Bedrohungsszenarios nutzt. Egal, ob die Fremdgeräusche nun schwächer oder stärker werden oder ob sie möglicherweise gar keine Fremdgeräusche sind: Jede nur denkbare Wendung ist nichts anderes als eine weitere Drehung der Schraube in Richtung einer unentrinnbaren Zwangsläufigkeit. Anders formuliert, zeigt Kafkas Der Bau in brillanter Folgerichtigkeit und mit immer neuen Anläufen die Unmöglichkeit, die Perspektive einer Beobachtung zweiter Ordnung einzunehmen, solange man ein Subjekt ist und nichts weiter.

"Es ging so weit daß ich manchmal den kindischen Wunsch bekam überhaupt nicht mehr in den Bau zurückzukehren sondern hier in der Nähe des Eingangs mich einzurichten, mein Leben in der Beobachtung des Eingangs zu verbringen und immerfort mir vor Augen zu halten und darin mein Glück zu finden, wie fest mich der Bau, wäre ich darin, zu sichern imstande wäre" (Kafka 1994: 178).

Das Glück des Wohnens in sicherer Geborgenheit, es würde dem Baumeister und Nutznießer dieser aufwendigen Anlage paradoxerweise erst und nur dann zuteil, wenn er dauerhaft darauf verzichtete, es in Anspruch zu nehmen. Allein im Beobachterstatus kann sich die Ausführung des Lebens ihrer Sache sicher sein, um den Preis, sie dadurch aber definitiv zu verlieren.

\section{Musil Und dAs GeschehenLassen}

Robert Musils Roman-Protagonist, der Mann ohne Eigenschaften, hieß in einer der Vorstufen noch anders, nämlich Anders. Darin enthalten ist die philosophische Grundformel der Kontingenz, nämlich das Bewusstsein, es könnte »ebensogut anders sein«, wie Ulrich das später formulieren wird. Ulrich und sein Roman handeln nach dem »Prinzip des unzureichenden Grundes«, demzufolge just das geschieht, »'was eigentlich keinen rechten Grund hat«« (Musil 1978: 13). Der Vorgänger-Figur ist diese Erkenntnis gleichsam auf den Leib geschrieben; den heranwachsenden Ulrich hatte sie bei manchen gesellschaftlichen Einrichtungen noch heftig anecken lassen, etwa in der Schule oder bei den sogenannten Wür- 
denträgern. Dann aber macht Ulrich seinen Frieden mit dem unzureichenden Grund des Wirklichen und lässt sich vom Sosein eines Weltlaufs dahintragen, in welchem nur »seinesgleichen « geschieht und es nicht angeraten scheint, länger auf das Richtige oder Eigentliche zu warten. Vor die Frage gestellt, wie er sein nach längerer Auslandsabwesenheit in Wien neu bezogenes Domizil ausgestalten solle, entscheidet sich Ulrich, offenbar durch finanzielle Rücksichten nur wenig eingeschränkt, für einen munteren Eklektizismus, indem er die Auswahl stilistischer Noten den Innenausstattern und Handwerkern überlässt.

Und auch das Gebäude selbst, ein großzügiges, die Baustile mehrerer Jahrhunderte in sich vereinigendes Schlösschen, bringt in der Ungleichzeitigkeit seiner architektonischen Prägungen die Haltung eines fast indifferenten Gewährenlassens zum Ausdruck, die sein neuer Besitzer sich während seines einjährigen Urlaubs vom Leben zu eigen macht.

"[W]enn man an seinem schmiedeeisernen Gitter vorbeikam, so erblickte man zwischen Bäumen, auf sorgfältig geschorenem Rasen etwas wie ein kurzflügeliges Schlößchen, ein Jagd- oder Liebesschlößchen vergangener Zeiten. Genau gesagt, seine Traggewölbe waren aus dem siebzehnten Jahrhundert, der Park und der Oberstock trugen das Ansehen des achtzehnten Jahrhunderts, die Fassade war im neunzehnten Jahrhundert erneuert und etwas verdorben worden " (Musil 1978: 12).

Indem diese Stadtvilla von einem zwar nicht allzu großen, aber durch Hecken wirkungsvoll abgesetzten, baumbestandenen Garten umgeben ist, vermag sie die Vorzüge einer parkartigen Anlage zu verbinden mit der eleganten Repräsentanz eines städtischen Ambientes. Denn auch die Fenster dieses Hauses bieten Straßenlage genug, um schauend und reflektierend am städtischen Leben und dem Gewimmel des Verkehrsstromes teilzuhaben.

Ulrich beschäftigt sich zu Beginn des Romans damit, zu sehen »wie ein Mann hinter einem Fenster « (ebd.: 259). ${ }^{7}$ Er ist getrennt von dem gesellschaftlichen Treiben, das er beobachtet, und bleibt in einem so be-

7 | Das noch in vielen weiteren Szenen eingesetzte Motiv des Fensters hat, wie Bernd-Rüdiger Hüppauf konstatierte, grundsätzlich "eine doppelte Wirkung", erzeugt trotz oder gerade mit der räumlichen Trennung zugleich eine optische Verbindung, "weil es die räumliche Trennung verwischt" (Hüppauf 1971: 28). 
tonten Sinne außenstehend, dass es naheliegt, jene den griechischen Skeptikern entlehnte phänomenologische Kardinaltugend der epoché, der affektneutralen Distanz des erkennenden Subjekts, in dieser Haltung wiederzuerkennen. ${ }^{8}$ Der gesellschaftliche Status und die selbstgewählte Funktion Ulrichs finden in seiner Lage und Haltung eine sinnfällige Verdichtung; mit seinen weithin treffenden, geradezu durchbohrenden Blicken ist er zwar mittendrin im Geschehen, und doch verfolgt er es mit stiller Reserve. Der Protagonist agiert in methodisch kontrolliertem Sinne als ein teilnehmender Beobachter; stets bedacht auf seinen privaten, inkommensurablen Rückzugsraum. Der Standpunkt ist derjenige eines stets auf der Schwelle zum gesellschaftlichen Leben verharrenden Betrachters, eines Intellektuellen, der das Leben vorübergleiten lässt, ohne sich an ihm festzukrallen.

In rückwärtiger Ausrichtung schwebt das Anwesen in einer fast meditativen Ruhe (wie sie die »Atemzüge eines Sommertags«, Musils letztes fertiggestelltes Kapitel, schildern), nach vorn dagegen herrscht großstädtische Betriebsamkeit. Die Diskrepanz von Gartenseite und Hauptfront entspricht der charakterlichen Doppelnatur des Protagonisten, von der eine frühe Skizze festhält:

"Schon in jeder Schilderung: Er ist groß, blond, breit, schmalhüftig... - bei D. klein, winzig. - Am Polizeirevier usw. Je nach Stimmung. Sein Haus ist bald ein Palais, bald ein Gartenhaus. Er denkt so oder entgegengesetzt über die gleiche Sache. [...] Er sieht die Welt muschelartig. D. h.: die Welt ist jetzt eine Muschel" (Musil 2010: Nachlass-Mappe 2/04/043).

Das entscheidende Merkmal der Muschel ist ihr an der Grenze von Organischem und Anorganischem wie ein kristallines Artefakt ausgebildetes Gehäuse. Zwischen dem Habitus der am Körper getragenen Kleidung und der Behausung, die aus den Mauern des Gebäudes besteht, braucht die Muschel sich nicht zu entscheiden.

Für die Ausbildung moderner Subjektivität kann sie insofern als modellhaft gelten, als sich in der Lebensstruktur der Muschel formbare Weichheit und stabile Festigkeit als zwei einander sonst ausschließende

8 | Vgl. Menges 1976: 140; die theoretischen Affinitäten Musils zur Phänomenologie Husserls untersucht Cellbrot 1988; zum Stichwort der "epoché" vgl. auch Musils Exzerpte in Heft 34 (Musil 1983: 887). 
Eigenschaften verbinden. Es war tatsächlich ein bestimmtes Bauwerk, bei dem Musil die Synthese von Gartenpark und Straßenfront in vergleichbarer, muschelartiger Synthese verwirklicht sah, nämlich das im dritten Bezirk an der Rasumofskygasse gelegene Palais Salm. Lange bevor der Schriftsteller in den 1920er Jahren selbst in der Rasumofskygasse eine Wohnung bezog (selbstredend nicht in dem prächtigen Palais, aber mit der Aussicht darauf aus seinem Arbeitszimmer), war Musil bei einem seiner Spaziergänge durch die äußeren Bezirke Wiens auf die Besonderheit jenes baulichen Ensembles aufmerksam geworden und hatte sie in seinem Tagebuch in skizzenartiger Beschreibung festgehalten.

"Form eines Platzes: Die Straße ist irgendwann einmal aufgeschüttet worden. So steckt das alte Palais mit den Tiefparterrefenstern, teils halb, teils ein Viertel, teils weniger in der Erde. An der Seitenfront wölbt sich hinter einem Gitter ein Säulenportal hinunter zu einem verwahrlosten Garteneck, od. Hofeck unter dem Niveau der Straße" (Musil 1983/1: 275).

Hier scheint in der Tat das Vorbild jenes Domizils porträtiert zu sein, das im Roman als »ein vor den Toren der Stadt liegender Sommersitz« vorgestellt wird, »der seine Bestimmung verlor, als die Großstadt über ihn wegwuchs « ${ }^{9}$ - fasst doch die Eintragung mit der Anhebung der Straße genau den Vorgang ins Bild, mit dem die Großstadt und ihr Verkehrsraum hinwegwachsen über ein Garteneck, das als verwahrlost bezeichnet wird, somit gleichfalls seiner Bestimmung verlustig ging. Musil zog 1921 in das Haus in der Rasumofskygasse 20 ein, auf die gegenüberliegende Seite jenes in Heft 7 skizzierten Anwesens. ${ }^{10}$ Die Beschreibung der »Form eines Platzes« aber erfolgte bereits sieben Jahre zuvor, am 7. August 1913, mit anderen Worten: an einem »Augusttag des Jahres 1913“ (Musil 1978: 9). In die Häuser- und Straßenskizzen dieses Heftes trug Musil nachträglich eine Notiz zu deren literarischer Verwertung ein (vgl. Musil 1983/1: 274), die nach Frisé als »erster Hinweis auf den frühen Roman-Entwurf

9 | Musil 1978: 13; vgl. Honold 1995: 120 u. Mülder-Bach 2013: 9.

10 | Zur Zeit dieser Eintragung wohnte Musil gleichfalls im "Landstraßen “-Bezirk, und zwar "im 3. Stock des damals neuen und modernen Hauses Untere Weißgerberstraße 61 in der Nähe der Rotundenbrücke" (Dinklage 1960: 223; vgl. auch Musil 1983/1: 251 u. Musil 1983/2: 152, Anm. 2). 
Spion «11 und damit als Keimzelle des Romanprojekts Mann ohne Eigenschaften $\mathrm{zu}$ betrachten ist. Noch weitere Indizien sprechen dafür, dass sich der spätere Schauplatz des Romans nicht nach dem naheliegenden Blick aus dem Arbeitszimmer richtete, sondern umgekehrt Musil die Nähe jenes Platzes suchte, an dem sich entscheidende Raumvorstellungen des fiktionalen Entwurfs bereits angelagert hatten. Da ist zunächst die große Radialachse, die aus der Inneren Stadt auswärts führt und die das anomische Geschiebe des Verkehrs, wie es von der Eingangsszene des Romans in seinem unkontrollierbaren rhythmischen Pulsieren evoziert wird, einer Art Flussbett gleich aufnimmt und in die flächige Weite der Peripherie weiterträgt.

Genau an der (nachmals) eigenen Straßenecke erfasst Musils frühe Stadterkundung vom Sommer 1913 metaphorisch die Großstadtbewegungen der vorüberziehenden Massen als ein quasi-hydrographisches Strömungsverhalten:

"Landstraße bei der Einmündung der Rasumofskygasse. Sie erweitert sich wie ein Fluß zu einem kleinen See stagniert u. dann in zwei Rinnsalen abfließt [...] Eine Insel mit braunen Holzhütten u. zwei Reihen Bäumen, von einer Querstraße durchschnitten, nahe an das eine Ufer gerückt» (Musil 1983/1: 275).

Der Skizzierung dieses Straßenverlaufs kommt, nicht allein wegen der Rekonkretisierung des habitualisierten metaphorischen Sprachgebrauchs vom Fluss des Verkehrs, eine poetologische Bedeutung zu. Die Betrachtung steht in Musils Aufzeichnungen in unmittelbarer Nähe zur Beschreibung des Palais Salm, so wie jenes sich auf dem Stadtplan in direkter Nachbarschaft der hier angesprochenen Straßenecke befindet. Musil fertigte im August 1913 sowohl mit diskursiven wie auch mit grafischen Mitteln die Skizze eines architektonischen Ensembles an, das ihm nach dem Kriege (oder vielmehr: zwischen den Kriegen), d. h. in den gut anderthalb Jahrzehnten der Wiener Schreibzeit am Mann ohne Eigenschaften, dann beim Blick aus dem Fenster vor Augen stehen wird.

Die Ecke von Rasumofsky- und Salmgasse im August 1913 ist der Chronotopos des noch ungeschriebenen Romans. Vor allem aber fand Musil genau hier die besagte Dualität von Straßen- und Gartenwelt vor, die über

11 | Musil 1983/2: 167, Anm. 45 (Kommentar des Hg.). 
die Phasen der frühen Entwürfe hin bis in die endgültige Romanfassung beibehalten und ausgebaut wurde:

"Viele dieser alten Häuser haben Gärten hinter sich. Man blickt durch eine Einfahrt auf einen kleinen steingepflasterten Hof, dann ein Gitter und ein paar Stufen erhöht ein Garten mit dunkelgrünen Baumkugeln. Oder man sieht das durch ein halbgeöffnetes hinteres Tor, dessen andre Hälfte braungelbe und blaue Scheiben hat, die einen Stern oder ein Kreuz bilden“ (ebd.).

So genau sich die Beschreibung an die Einzelheiten des Wahrgenommenen hält, so unverkennbar wird dem durch den Blick abgetasteten Raumprofil ein Muster unterlegt, das bereits die wesentlichen Strukturachsen der Darstellung von Ulrichs Schlösschen präformiert. Da ist zunächst die Polarität der Blickrichtungen, auf welche die Angaben zu Art und Umständen des Einblick-Nehmens die Aufmerksamkeit lenken. Während der Blick von der Straßenseite dem für Besucher vorgesehenen Zugang durch die Einfahrt folgt, vermittelt die Rückseite die weniger offizielle Ansicht, die dem Betrachter ein »halbgeöffnetes hinteres Tor« gewährt. Auch das im Nachlasskapitel »Sonderaufgabe eines Gartengitters« (Musil 1978: 1405) und in dessen Varianten zentrale Raumelement des Gitters, das als einseitig durchlässiger Filter des Blicks Großstadtwelt und Gartenenklave zugleich trennt und verbindet, wird in der Skizze festgehalten.

Betont die horizontale Raumachse mit dem Innen/Außen-Gegensatz die Dualität von öffentlichem und privatem Raum, so wird diese synchrone Spannung durch eine zweite, vertikale Achse als Resultat einer diachronen Abfolge gesellschaftlicher Formationen interpretiert, deren Spuren sich schichtweise abgelagert haben (das »Garteneck [...] unter dem Niveau der Straße«, die »irgendwann einmal aufgeschüttet« wurde (Musil 1983/1: 275)). Entlang dieser beiden Achsen des Blicks wird die Beschreibung des Anwesens in der Salmgasse vermessen, das die Merkmale der Ambivalenz von Schau- und Rückseite wie die der historischen Mehrschichtigkeit in prototypischer Weise in sich vereinigt. »Das große Tor in der Salmgasse: es nimmt fast ein Viertel der Front des kleinen Hauses ein. Es ist immer zur Hälfte offen; man sieht einen von einer Seite zur andern ansteigenden Hof, mit runden Steinen gepflastert, Gras dazwischen« (Musil 1983/1: 274; Eintragung vom 4. August 1913). Die Druckfassung wird später den hier nur angedeuteten Gedanken der Darstellung von Geschichte im etymologisch prägnanten Sinne einer Abfolge von 
Schichten bis zur grotesken Überspitzung ausfabulieren. Gilt es dabei, den sich einem überraschten Spaziergänger tout à coup bietenden Anblick eines prunkvollen Gartenschlösschens inmitten großstädtischer Szenerie in sukzessive Narration zu übersetzen, so ist bemerkenswert, in welche Abfolge die Raumelemente vom Erzähldiskurs gebracht werden. Einer »spontanen« Beschreibung des Anwesens auf der Ebene Garten/Gitter/ Straße folgt das Kippen dieser Achse in die Vertikale und damit die historisch informierte Aufmerksamkeit für die Sequenz von Stilrichtungen, die an diesem Palais verbaut wurden.

»Haus und Wohnung des Mannes ohne Eigenschaften«, so das zweite Kapitel des Romans, werden von der inneren Stadt aus erreicht durch einen »jener langen, gewundenen Verkehrsflüsse, die strahlenförmig am Kern der Stadt entspringen, die äußeren Bezirke durchziehen und in die Vorstädte münden« (Musil 1978: 11). Im Duktus der Erzählung wird ein ebensolcher Fluss gebildet, der aus dem Porträt der Reichshaupt- und Residenzstadt Wien (und damit aus dem historisch-gesellschaftlichen Allgemeinen) zur fiktiven Person des Protagonisten Ulrich (und damit zum Besonderen des Einzelfalles) weiterführt, voranschreitend wie im Sog des beschriebenen städtischen Verkehrsflusses. Ein Unfall war geschildert worden, aus dem bemerkenswerterweise nichts hervorging, außer vielleicht dem flüchtigen Blick auf die verstörte Reaktion eines Passantenpaares, das stadtauswärts flanierte, ebenfalls vom Verkehrsfluss getragen. »Sollte ihm das elegante Paar noch eine Weile weiter gefolgt sein, so würde es etwas gesehen haben, das ihm gewiß gefallen hätte« (Musil 1978: 11). Nämlich das eindrucksvolle, Stadtsitz und Landhausstil so ungewöhnlich miteinander verbindende Domizil des Roman-Protagonisten, auf dessen nähere Lebensumstände und Charakterisierung der Erzählvorgang nach dieser Überleitung dann einschwenken kann.

Am Puls ihres Verkehrs und am Gang ihrer Geschäfte sei eine Stadt zu erkennen, so heißt es; mit der fließenden Bewegung der Straße nimmt auch die Erzählung merklich Fahrt auf. Der Mann am Fenster, von dem schon die Rede war, Ulrich also,

"zählte mit der Uhr seit zehn Minuten die Autos, die Wagen, die Trambahnen und die von der Entfernung ausgewaschenen Gesichter der Fußgänger [...]; er schätzte die Geschwindigkeiten, die Winkel, die lebendigen Kräfte vorüberbewegter Massen, die das Auge blitzschnell nach sich ziehen, festhalten, Ioslassen, die während einer Zeit, für die es kein Maß gibt, die Aufmerksamkeit zwingen, sich gegen sie zu 
stemmen, abzureißen, zum nächsten zu springen und sich diesem nachzuwerfen“ (Musil 1978: 12).

Musils Protagonist betreibt ein Wahrnehmungsexperiment mit der transitorischen Rapidität der Großstadt, und gleichzeitig führt der Erzähler ein Experiment mit der Erzählbarkeit singulärer Geschichten im Zeitalter rasch bewegter Massen durch. Einzelnes herauszugreifen und sich daran mit Aufmerksamkeit länger als einen Augenblick zu heften, ist eine Herausforderung, die nur gegen den Sog des Verschwindens durchzusetzen ist. Dem endlosen Strom, der eindruckslosen Vielzahl und Flüchtigkeit von Sensationen kann nur mit einer gewissen Willkür überhaupt noch etwas Besonderes entnommen werden; und schon gar etwas, was über längere Strecken der Beachtung wert wäre. Was gelten Einzelschicksale im Zeitalter von Statistik, Masse und Markt? Dabei ist es gerade dieses scheinbar chaotische Gewusel des städtischen Verkehrs, an welchem die heroische Kraft eines zeitgenössischen Helden sich messen lassen müsste. Ulrich stellt nach seinen Beobachtungen die folgende Überlegung an:

"Könnte man die Sprünge der Aufmerksamkeit messen, die Leistungen der Augenmuskeln, die Pendelbewegungen der Seele und alle die Anstrengungen, die ein Mensch vollbringen muß, um sich im Fluß einer Straße aufrecht zu halten, es käme vermutlich - so hatte er gedacht und spielend das Unmögliche zu berechnen versucht - eine Größe heraus, mit der verglichen die Kraft, die Atlas brauchte, um die Welt zu stemmen, gering ist, und man könnte ermessen, welche ungeheure Leistung heute schon ein Mensch vollbringt, der gar nichts tut“ (Musil 1978: 12).

Mittlerweile sind solche Kognitionsleistungen bzw. deren neuronale Infrastruktur sehr gut messbar geworden, im Ansatz war das schon in der von Musil studierten empirischen Psychologie der Berliner Schule der Fall. Entscheidend aber an diesen Überlegungen ist, dass in ihnen die Leistung des Individuums gekoppelt wird an seine Auseinandersetzung mit einer Lebenswelt, welcher die anthropozentrischen Gewissheiten längst abhandengekommen sind.

Filter und Netz des Fenstergitters stellen an der Schwelle von In- und Exklusion jene künstlichen Vorkehrungen her, die den bloßen Blick auf die Straße erst zum Experiment werden lassen - Distanz und Segmentierung. Die Subjektivität des Beobachters überlebt in dieser satirischen Nachstellung des naturwissenschaftlichen Experimentierens allein durch 
seine Macht zur perspektivischen Verkürzung; die souveräne Eingrenzung des observierten Gebietes erlaubt es ihm, das Straßenleben demonstrativ am Rande des Blickfelds enden zu lassen, wiewohl es, unbekümmert um den optischen Zugriff des Mannes am Fenster, auch jenseits dieses Rahmens gewiss seinen Fortgang nimmt. Ulrich, der Mann ohne Eigenschaften, erlangt somit eine spezifisch kakanische Form der Dominanz über die urbane Mitwelt: nämlich die scheinbar vollständige Kontrolle über das, was ohnehin geschieht.

\section{LITERATURVERZEICHNIS}

Blumenberg, Hans ( ${ }^{6} 2001$ [1979]): Arbeit am Mythos, Frankfurt a. M.

Cellbrot, Hartmut (1988): Die Bewegung des Sinnes. Zur Phänomenologie Robert Musils im Hinblick auf Edmund Husserl, München.

Dinklage, Karl (1960): »Musils Herkunft und Lebensgeschichte«, in: Ders. (Hg.): Robert Musil. Leben, Werk, Wirkung, Reinbek b. Hamburg, S. 187-264.

Encke, Julia (2006): Augenblicke der Gefahr. Der Krieg und die Sinne. 1914-1934, Paderborn.

Haag, Saskia (2012): Auf wandelbarem Grund. Haus und Literatur im 19. Jahrhundert, Freiburg i. Br.

Heidegger, Martin (2000 [1951]): »Bauen Wohnen Denken«, in: Ders.: Gesamtausgabe. I. Abteilung: Veröffentlichte Schriften 1910-1976. Bd. 7, Vorträge und Aufsätze, hg. v. Friedrich-Wilhelm von Hermann, Frankfurt a. M., S. 145-164.

Honold, Alexander (1995): Die Stadt und der Krieg. Raum- und Zeitkonstruktion in Robert Musils Roman »Der Mann ohne Eigenschaften«, München.

Hüppauf, Bernd-Rüdiger (1971): Von sozialer Utopie zur Mystik. Zu Robert Musils »Der Mann ohne Eigenschaften«. Musil-Studien Bd. 1, München.

Kafka, Franz (2002): Tagebücher, hg. v. Hans-Gerd Koch/Michael Müller/Malcolm Pasley, Heft 11, Frankfurt a. M.

Kafka, Franz (1994): »Ich habe den Bau eingerichtet...«, in: Ders.: Gesammelte Werke in zwölf Bänden, hg. v. Hans-Gerd Koch. Bd. 8, Frankfurt a. M., S. 165-208. 
Kittler, Wolf (1990): »Grabenkrieg - Nervenkrieg - Medienkrieg. Franz Kafka und der 1. Weltkrieg«, in: Jochen Hörisch/Michael Wetzel (Hg.): Armaturen der Sinne. Literarische und technische Medien 1870-1920, München, S. 289-309.

Menges, Karl (1976): »Robert Musil und Edmund Husserl: Über phänomenologische Strukturen im >Mann ohne Eigenschaften« «, in: Modern Austrian Literature 3/4, S. 131-154.

Menke, Bettine (1992): »Aufgegebene Lektüre: Kafkas >Der Bau«, in: Ludo Verbeeck (Hg.): Die Aufgabe des Lesers, Leuven, S. 147-175.

Mülder-Bach, Inka (2013): Robert Musil. Der Mann ohne Eigenschaften. Ein Versuch über den Roman, München.

Musil, Robert (1978): Der Mann ohne Eigenschaften, hg. v. Adolf Frisé, Reinbek b. Hamburg.

Musil, Robert (1983): Tagebücher, 2 Bde., hg. von Adolf Frisé, neu durchges. und erg. Auflage, Reinbek b. Hamburg.

Musil, Robert (2010): Kommentierte digitale Edition sämtlicher Werke, Briefe und nachgelassener Schriften. Klagenfurter Ausgabe, hg. von Walter Fanta/Klaus Amman/Karl Corino, Klagenfurt.

Steiner, Uwe C. (2012): Ohrenrausch und Götterstimmen. Eine Kulturgeschichte des Tinnitus, München. 